\title{
Expression and subcellular localization of HSPC117 in min pig tissues and the PK15 cell line
}

\author{
Hong $\mathrm{Ma}^{1}$, Bo Fu${ }^{1}$, Xu Zhang, Liang Wang, Zhongqiu Li and Di Liu* \\ Heilongjiang Academy of Agricultural Sciences, Harbin, Heilongjiang, China
}

\begin{abstract}
.
BACKGROUND: The human hematopoietic stem/progenitor cell 117 (HSPC117) protein is involved in many important biological processes.

OBJECTIVE: This study was designed to identify the level of HSPC117 mRNA expression in 10 min pig tissue samples and HSPC117 subcellular localization in the PK15 cell line.

METHODS: In this study, 10 tissue samples of min pigs were collected, and EGFP-HSPC117 vectors were constructed to express EGFP-HSPC117 fusion proteins in PK15 cells.

RESULTS: HSPC117 mRNA was expressed in all of the tissue samples, although the levels of expression in fat and lung tissues were significantly lower than in other tissues $(P<0.01)$. After generating and detecting the EGFP-HSPC117 fusion protein, fluorescence was found to be distributed throughout the cytoplasm and nucleus during interphase; however, the fluorescence was concentrated in the nuclear area in mitotic cells.

CONCLUSIONS: These results indicate that the HSPC117 gene is expressed in many min pig tissues. The HSPC117 protein was distributed throughout the cells during interphase, but was concentrated in the nuclear area in mitotic cells.
\end{abstract}

Keywords: HSPC117, tissue expression, subcellular localization

\section{Introduction}

The human hematopoietic stem/progenitor cell 117 (HSPC117, Synonyms C22 or f28, FAAP) protein is an important component of protein complexes. For example, HSPC117 is present in the osmotic response element binding protein KIAA0827 and TNF- $\alpha$ mRNA 3' AU-rich element binding complexes [1]. Previous studies have shown that a homologous protein of HSPC117 also interferes with the activation of mitogen-activated protein kinase (MAPK), activates the serum response factor (SRF), and influences embryonic and placental development [2-4]. It has also been found in the spreading initiation center (SIC). HSPC117 can also bind to talin and vinculin [5]. The expression of HSPC117 is activated by agents and environmental pressure, which impair the translation apparatus [6]. The cold environment can disrupt the gene transcription process in the cell, while HSPC117 is involved in helping the cell to survive the translation apparatus. All of these findings indicate that HSPC117 may be

\footnotetext{
${ }^{1}$ These authors contributed equally to this work and should be considered co-first authors.

${ }^{*}$ Corresponding author: Di Liu, Heilongjiang Academy of Agricultural Sciences, Harbin, Heilongjiang, China. Tel.: +86 451 8667 7458; E-mail: liudi1963@163.com.
}

0928-7329/19/\$35.00 (C) 2019 - IOS Press and the authors. All rights reserved

This article is published online with Open Access and distributed under the terms of the Creative Commons Attribution NonCommercial License (CC BY-NC 4.0). 
involved in many important functions. The expression profile of HSPC117 has been studied in tissues of Meishan, Large White, and Meishan/Large White hybrid pigs. HSPC117 gene expression was found in many tissues and is believed to influence the hybrid phenotype [7]. The min pig is a local pig living in northeastern China, which adapts to the cold climate in this area. HSPC117 is an important protein, but the expression and subcellular localization of HSPC117 in min pig tissues have not yet been elucidated.

Here, HSPC 117 mRNA expression features are characterized in different tissues from the min pig, reflecting the physiological function of the gene. The subcellular localization of HSPC117 is also examined in porcine kidney epithelial cells (PK15) during interphase and mitosis, indicating the region in which the protein plays a role. This work may be able to indicate parts of the mechanism underlying cold resistance in min pigs.

\section{Materials and methods}

\subsection{Animal}

Three female min pigs were studied. The min pigs were slaughtered at 8 months of age and samples of back muscle, leg muscle, kidney, spleen, fat, uterine, small intestine, lung, and oviduct were collected.

\subsection{Reverse transcription and real-time quantitative PCR (RT-qPCR)}

Total RNA was extracted from each sample using a RNA extraction kit. The purity and integrity of the total RNA were assessed. The qualified sample total RNAs were used for reverse transcription. The first-strand cDNA was synthesized using an M-MLV First Strand Kit (Invitrogen) with oligo-dT as a primer according to the manufacturer's protocol.

Gene expression was quantified using the Stratagene Mx3000P qPCR system. Glyceraldehyde3-phosphate dehydrogenase (GAPDH) mRNA levels were measured in all of the tissue samples to normalize all of the mRNA quantifications. The specific primers used in these tests included GAPDH (F, 5'ACACTCACTCTTCTACCTTTG3'; R, 5' CAAATTCATTGTCGTACCAG3'), HSPC117 (F, 5'TCCCATAGTAACGCCAATA 3'; R, 5'CTGGGTGTCCACAATCAA3'). All of the PCR amplifications were performed in triplicate independent biological replicates ( 3 samples of each kind of organ); no template controls were included. The relative amount of mRNA to GAPDH in each sample was calculated using a relative quantification standard curve.

\subsection{Construction of EGFP-C1-HSPC117 expression plasmid}

The porcine HSPC117 coding sequence (GenBank: DQ 508263.1) was amplified by PCR from PK15 cell RT-PCR products using specific primers containing restriction sites at $5^{\prime}$ ends. $\mathrm{F}$ ( $5^{\prime}$ AAGCTTATGA GTCGCAGCTATAATGATGAG3') and R (5'GGATCCCTATCCTTTGATCACAGCAATTGGTC $3^{\prime}$ ).

The PCR product was cloned into pEGFP-C1 vector at HindIII/BamHI sites to construct pEGFP-C1HSPC117 (EGFP-HSPC117) and fully sequenced.

\subsection{Cell lines, cultures, and transfections}

The PK15 cells were cultured in DMEM supplemented with $10 \%$ fetal calf serum at $38^{\circ} \mathrm{C}$ in a humidified incubator $\left(5 \% \mathrm{CO}_{2}\right.$ and $95 \%$ air). Prior to the day of transfection, the fusion rate of PK15 cells had reached approximately $80-90 \%$ and the PK15 cells were plated into $35-\mathrm{mm}$ plates. EGFP-HSPC117 was 


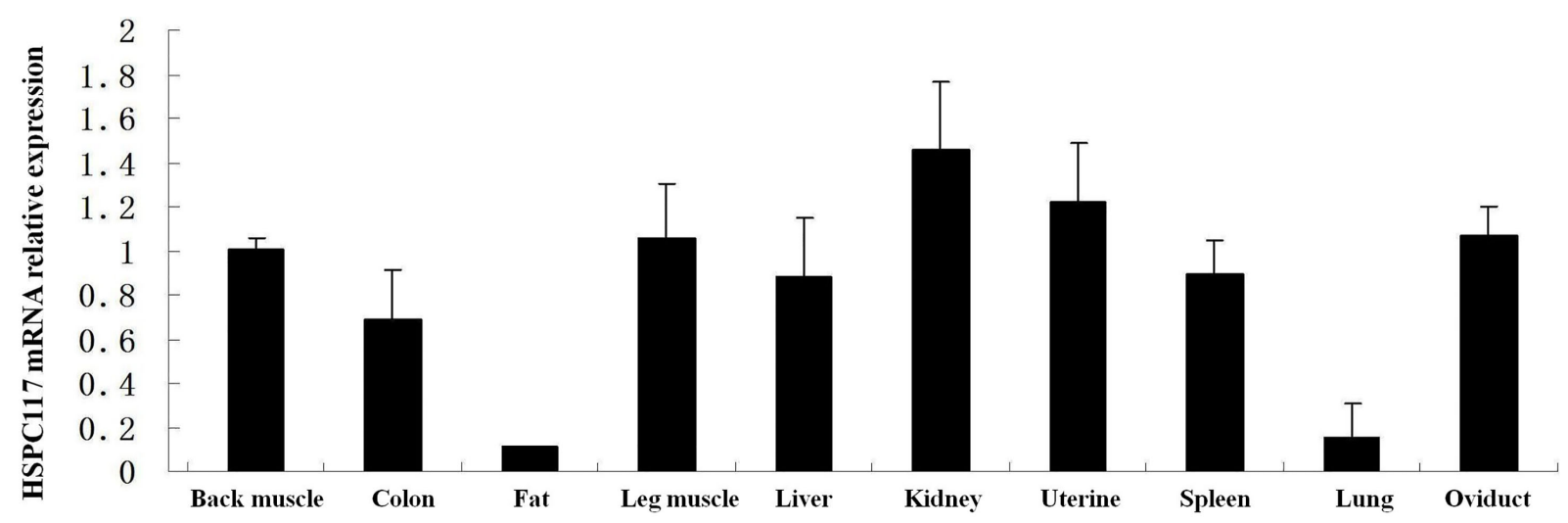

Fig. 1. The HSPC117 mRNA expression level in samples of 10 types of tissue (back muscle, colon, fat, leg muscle, liver, kidney, uterus, spleen, lung, and oviduct) GAPDH was used as the control.

transiently transfected in PK15 cells using $10 \mu \mathrm{L}$ Lipofectamine 2000 reagent and $7 \mu \mathrm{g}$ EGFP-HSPC117 plasmid, according to the reagent's protocol. The PK15 cells were cultured in the transfection medium for $6 \mathrm{~h}$ and then incubated in DMEM medium with $10 \%$ serum.

\subsection{Analysis of fluorescence images}

The transfected PK15 cells were cultured for 24 hours, 48 hours and 72 hours. Prior to observation, the cells were stained with Hoechst 33342 for $10 \mathrm{~min}$. An Axioimager fluorescence microscope (Zeiss) with a $20 \times$ or a $40 \times$ objective and Axiovision imaging software (Zeiss) was used for imaging. GFPHSPC117 and Hoechst 33342 fluorescence analyses were conducted using the GFP (488-nm) and DAPI (340-nm) channels, respectively.

\subsection{Statistical analysis}

Data assays were performed using one-way ANOVA using SPSS 15.0 software. All of the data are presented as mean \pm SEM. Moreover, $P<0.05$ was considered to be statistically significant. Three replicates were analyzed for each experiment.

\section{Results and discussion}

\subsection{Relative expression of HSPC117 mRNA in tissues}

To determine the relative expression of HSPC117 mRNA in tissues from the min pig, qPCR was used to analyze mRNA levels in samples of 10 types of tissue. As shown in Fig. 1, the HSPC117 mRNA expression levels in most of the tissues (back muscle, colon, leg muscle, liver, kidney, uterus, spleen, and oviduct) were similar $(P>0.05)$. However, the HSPC117 mRNA expression levels in fat and lung tissues were a significant 0.12 and 0.19 times lower, respectively, compared than in back muscle $(P<$ $0.01)$.

The HSPC117 protein is involved in many important cell functions, such as tRNA splicing, RNA transportation, cell migration/invasion, and many other essential functions, which occur during cell growth 

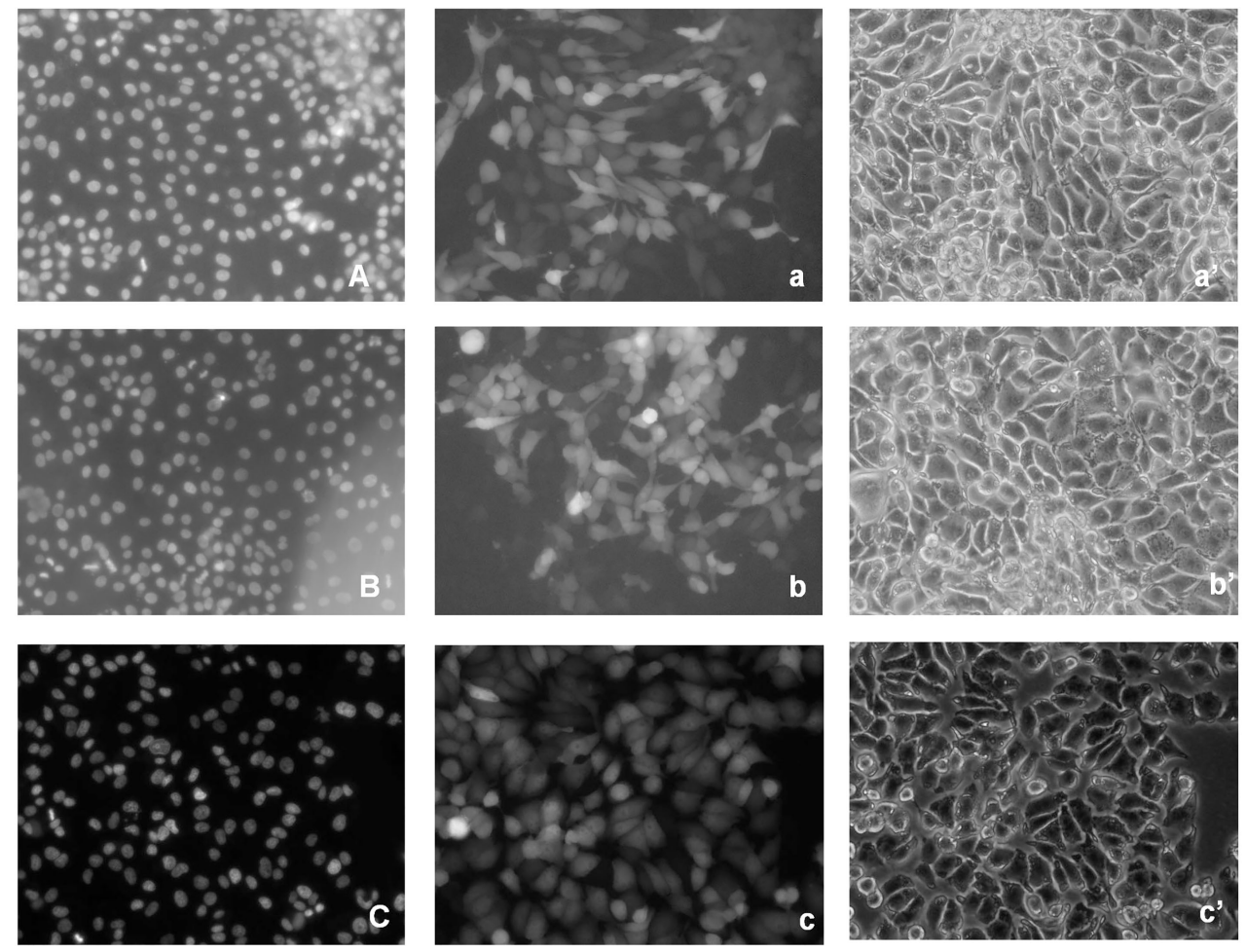

Fig. 2. EGFP-HSPC117 localization in PK15 cells during interphase. A: 24 h nucleus. a: 24 h EGFP-HSPC117. a': 24 h contrast image. B: 48 h nucleus. b: 48 h EGFP-HSPC117. b': 48 h contrast image. C: 72 h nucleus. c: 72 h EGFP-HSPC117 fluorescence. c': $72 \mathrm{~h}$ contrast image.

and division [8]. Since the HSPC117 protein plays so many basic and important roles in the biological processes of cells, it is widely distributed in both cells and tissues. Previous research has demonstrated that the HSPC117 protein is expressed in tissues from humans and in some types of pigs $[8,9]$. The D10Wsu52e protein (an HSPC117 homolog) has been found in mouse embryonic forebrain, midbrain, hindbrain, optic vesicles, somites, stomach, and duodenum, and the expression level of this protein changes depending on the development stage [10]. In the current study, HSPC117 mRNA expression was found in back muscle, colon, fat, leg muscle, liver, kidney, uterus, spleen, lung, and oviduct tissues from the min pig. However, the level of HSPC117 mRNA expression differed among these tissues, suggesting that HSPC117 might have different functions or be regulated differently in different tissues, although it performs some basic and important biological functions.

\subsection{Subcellular distribution of the EGFP-HSPC117 protein in PK15 cells}

Transgenic PK15 cells, which express an EGFP-HSPC117 fusion protein, were produced. EGFPHSPC117 expression in the transgenic PK15 cells was driven by the SV40 CMVie promoter. We examined the distribution of the fusion protein in PK15 cells using fluorescence microscopy. EGFP fluorescence was visible in some PK15 cells $12 \mathrm{~h}$ after induction and was clearly seen in most cells $24 \mathrm{~h}$ after induction. Fluorescent fusion proteins were detected throughout the cytoplasm as shown by indirect fluorescence images, although EGFP expression was slightly stronger in cell nuclei than in the cytoplasm 

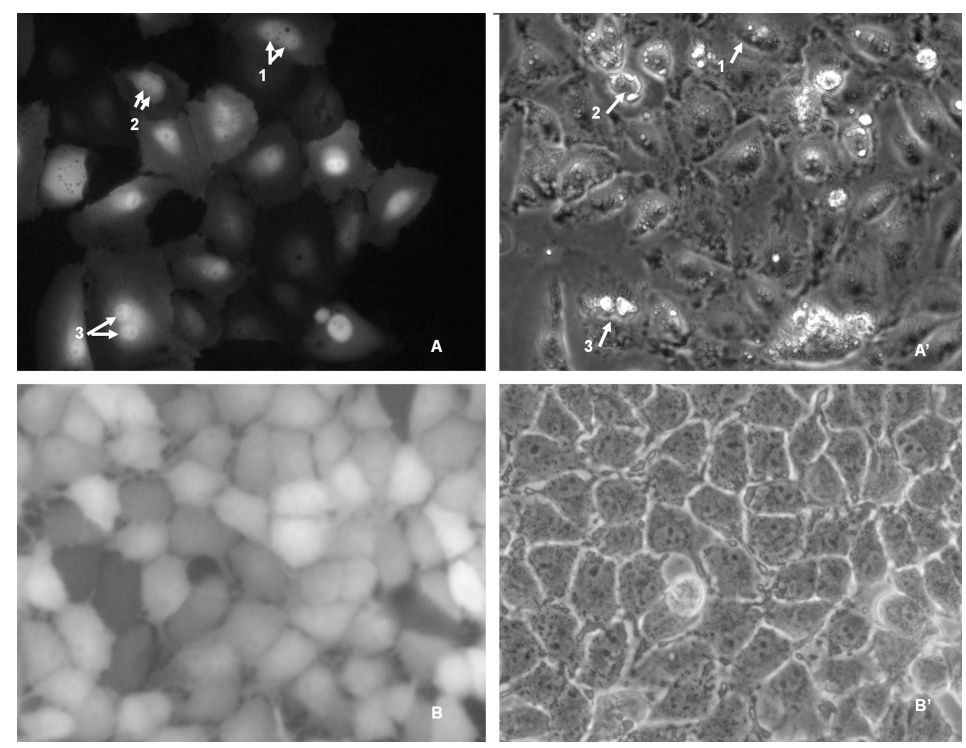

Fig. 3. EGFP-HSPC117 localization in PK15 cells during mitosis. A: The apparent EGFP fluorescence was detected in the dividing daughter nucleolus during mitosis. A' contrast image of PK15 cells during mitosis. B: fluorescent fusion proteins were detected throughout the cytoplasm during interphase. B' contrast image of PK15 cells during interphase.

(Fig. 2). There were no marked changes in green fluorescence during the interphase after the transgenic cells, expressing EGFP-HSPC117, were cultured for $48 \mathrm{~h}$ or $72 \mathrm{~h}$.

To elucidate the localization of EGFP-HSPC117 in PK15 cells during mitosis, transgenic PK15 cells were cultured for 3-4 days. Some cells were then observed during the mitotic phase. As shown in Fig. 3, fluorescence was detected in the condensed chromosomes of the mitotic PK15 cells, whereas much less fluorescence was seen in the cytoplasm. These observations indicate that the localization of EGFP-HSPC117 in PK15 cells changes during the mitotic phase.

HSPC117 is an important protein complex and it participates in various functions that occur in different parts of the cell. In the current study, EGFP-HSPC117 fusion protein fluorescence was detected throughout the cytoplasm and nuclei during interphase and the distribution did not change significantly over culture time. Perez-Gonzalez et al. found that HSPC117 protein molecules combined with DDX1, FAM98B, and hCLE proteins to form a compound, which is present in both the nucleus and cytoplasm and participates in both nuclear and cytoplasmic RNA metabolism [9]. Previous studies have shown that HSPC117 is present in the cytoplasm and is involved in both RNA maturation and transportation [11-13]. However, in this study, fusion protein fluorescence was visibly concentrated in the nucleolus area of PK15 cells during the mitotic phase. To the best of our knowledge, no previous research has indicated that HSPC117 assembles in the nucleolus area during the mitotic phase. This phenomenon might be related to chromosome condensation, although there is no direct evidence for this as of yet.

\section{Conclusions}

In the current study, HSPC117 mRNA expression levels were determined in samples of 10 types of tissue from the min pig (back muscle, colon, fat, leg muscle, liver, kidney, uterine, spleen, lung, and oviduct). In addition, the subcellular localization of HSPC117 was determined in the porcine kidney 
epithelial cell line (PK15). HSPC117 mRNA expression was detected in all 10 tissue types, however, the expression levels of HSPC117 mRNA in fat and lung tissues were significantly lower compared with the other 8 types of tissue. HSPC117-EGFP fusion protein fluorescence was distributed throughout the cytoplasm and nuclei during interphase, but was concentrated in the nuclear area in mitotic cells. Together, these results indicate that HSPC117 is an important protein that plays a role in nearly all tissues and in different stages of cell growth and development.

\section{Acknowledgments}

This work was supported by the Natural Science Foundation of Heilongjiang Province of China (JJ2018ZZ0082) and the National Natural Science Foundation of China (31872980). We thank LetPub (www.letpub.com) for its linguistic assistance during the preparation of this manuscript.

\section{Conflict of interest}

None to report.

\section{References}

[1] Simon J, Nick M, Mark P, David GC, Matthias G, Cohen P. Inhibition of SAPK2a/p38 prevents hnRNP A0 phosphorylation by MAPKAP-K2 and its interaction with cytokine mRNAs. The EMBO Journal. 2002; 21(23): 6505-6514.

[2] Heath RJ, Leong JM, Visegrady B, Machesky LM, Xavier RJ. Bacterial and host determinants of MAL activation upon EPEC infection: the roles of Tir, ABRA, and FLRT3. PLoS Pathogens. 2011; 7(4): e1001332.

[3] Wang Y, Hai T, Liu Z, Zhou S, Lv Z, Ding C, Liu L, Niu Y, Zhao X, Tong Ml. HSPC117 deficiency in cloned embryos causes placental abnormality and fetal death. Biochemical and Biophysical Research Communications. 2010; 397(3): 407-412.

[4] Ma H, Qi MY, Zhang X, Zhang YL, Wang L, Li ZQ, Fu B, Wang WT, Liu D. HSPC117 is regulated by epigenetic modification and is involved in the migration of JEG-3 cells. International Journal of Molecular Sciences. 2014; 15(6): 10936-10949.

[5] Hu J, Teng J, Ding N, He M, Sun Y, Yu ACH, Chen J. FAAP, a Novel Murine Protein, Regulates Cell Adhesion through Inhibiting Vinculin-Paxillin. Frontiers in Bioscience. 2008; 13: 7123-7131.

[6] Engl C, Schaefer J, Kotta-Loizou I, Buck M. Cellular and molecular phenotypes depending upon the RNA repair system RtcAB of Escherichia coli. Nucleic Acids Res. 2016; 44(20): 9933-9941.

[7] Xie HT, Lei MG, Xiong YZ, Deng CY, Jiang SW, Li FE, Zuo B, Xu DQ. Cloning and Identification of Porcine HSPC117 Gene Differentially Expressed in F1 Crossbreds and Their Parents, Agricultural Sciences in China. 2006; 38(11): 758758.

[8] Popow J, Englert M, Weitzer S, Schleiffer A, Mierzwa B, Mechtler K, et al. HSPC117 is the essential subunit of a human tRNA splicing ligase complex. Science. 2011; 331(6018): 760-764.

[9] Perez GA, Pazo A, Navajas R, Ciordia S, Rodriguez-Frandsen A, Nieto A. hCLE/C14orf166 associates with DDX1HSPC117-FAM98B in a novel transcription-dependent shuttling RNA-transporting complex. PloS one. 2014; 9(3): e90957.

[10] Zhao JD, Ju XF, Zhange LQ, Zhao Y, Liang Y, An TZ, Chun BT. Spatiotemporal expression of D10Wsu52e gene in the developing mouse stomach and duodenum. Acta Biophysica Sinica. 2011; 112(22): 755-764.

[11] Kellner JN, Reinstein J, Meinhart A. Synergistic effects of ATP and RNA binding to human DEAD-box protein DDX1. Nucleic Acids Research. 2015; 43(5): 2813-2828.

[12] Yoshihisa T. Handling tRNA introns, archaeal way and eukaryotic way. Frontiers in Genetics. 2014; 5: 213.

[13] Zebrowski DC, Vergarajauregui S, Wu CC, Piatkowski T, Becker R, Leone M, et al. Developmental alterations in centrosome integrity contribute to the post-mitotic state of mammalian cardiomyocytes. eLife. $2015 ; 6: 4$. 